Dementia

and Geriatric

Cognitive Disorders
Dement Geriatr Cogn Disord 2013;36:229-241

DOI: $10.1159 / 000351672$

Accepted: April 15, 2013

Published online: August 15, 2013

\title{
Long-Term Safety and Efficacy of Donepezil in Patients with Dementia with Lewy Bodies: Results from a 52-Week, Open-Label, Multicenter Extension Study
}

\author{
Manabu Ikeda ${ }^{a}$ Etsuro Mori ${ }^{b}$ Kenji Kosakac Eizo Iseki ${ }^{d}$ \\ Mamoru Hashimoto $^{a}$ Noriyuki Matsukawa $^{f}$ Kazutaka Matsuo $^{e}$ \\ Masaki Nakagawa ${ }^{\mathrm{e}}$ on behalf of the Donepezil-DLB Study Investigators \\ a Department of Neuropsychiatry, Faculty of Life Sciences, Kumamoto University, Kumamoto, \\ ${ }^{b}$ Department of Behavioral Neurology and Cognitive Neurosciences, Tohoku University \\ Graduate School of Medicine, Sendai, ' Department of Psychiatry, Yokohama City University \\ School of Medicine, Yokohama, 'd Juntendo Tokyo Koto Geriatric Medical Center, and 'Eisai Co., \\ Ltd., Tokyo, and fDepartment of Neurology, Nagoya City University, Nagoya, Japan
}

\section{Key Words}

Cholinesterase inhibitors · Cognitive fluctuations · Dementia with Lewy bodies · Donepezil

\begin{abstract}
Background/Aims: To investigate the safety and efficacy of long-term administration (52 weeks) of donepezil in patients with dementia with Lewy bodies (DLB). Methods: This was a 52-week, multicenter, open-label extension study. Up to 8 weeks after the completion of the preceding randomized, placebo-controlled trial (RCT), patients started treatment with $3 \mathrm{mg}$ of donepezil daily for 2 weeks, followed by $5 \mathrm{mg}$ daily for the remaining 50 weeks. Cognitive function, behavioral and psychiatric symptoms, cognitive fluctuations, and caregiver burden were assessed using the Mini-Mental State Examination, Neuropsychiatric Inventory, Cognitive Fluctuation Inventory, and the Zarit Caregiver Burden Interview, respectively. Safety parameters were monitored throughout. Results: In total, 108 patients were enrolled in the study. Cognitive function and dementia-related behavioral symptoms, including cognitive fluctuations, were improved after the start of donepezil treatment, and improvement was maintained for 52 weeks. Reduction in caregiver burden observed in the preceding RCT returned to the baseline level at 52 weeks. There was no significant imbalance in the incidence of adverse events (AEs) by onset time, and delayed AE onset induced by the long-term administration of donepezil was unlikely to appear. Conclusion: The long-term administration of donepezil at $5 \mathrm{mg} /$ day was well tolerated in patients with DLB and is expected to exhibit lasting effects, improving impaired cognitive function and psychiatric symptoms up to 52 weeks.




\begin{tabular}{l|l}
\hline Dement Geriatr Cogn Disord 2013;36:229-241 \\
\hline DOI: $\underline{10.1159 / 000351672}$ & $\begin{array}{l}\text { (c) } 2013 \text { S. Karger AG, Basel } \\
\text { www.karger.com/dem }\end{array}$ \\
\hline
\end{tabular}

Ikeda et al.: Long-Term Safety and Efficacy of Donepezil in Patients with Dementia with Lewy Bodies: Results from a 52-Week, Open-Label, Multicenter Extension Study

\section{Introduction}

Dementia with Lewy bodies (DLB) is a common form of dementia in the elderly, accounting for $10-15 \%$ of patients with dementia and constituting the second largest group after Alzheimer's disease (AD) [1]. The core clinical features of DLB include neuropsychiatric symptoms and parkinsonism, as well as cognitive impairment characterized by deficits in attention, executive function, and visual perception [2].

Compared with patients with $\mathrm{AD}$, cholinergic neurotransmission is more defective in patients with DLB [3]. In addition, although cholinergic losses in DLB affect both brainstem and basal forebrain presynaptic nuclei, postsynaptic cortical muscarinic and nicotinic receptors are functionally more intact [4]. For these reasons, it is suggested that cholinesterase inhibitors (ChEIs) may be effective for treating DLB. In fact, usefulness of ChEIs such as galantamine, rivastigmine, and donepezil in the treatment of DLB symptoms has been reported in several open-label studies [5-9] and two randomized controlled trials [10, 11]. Their usefulness has also been reported in several clinical trials of Parkinson's disease dementia, which is considered to fall into same category as DLB $[12,13]$. Recently, we reported in a 12-week, randomized, placebo-controlled trial (RCT) that donepezil at 5 and $10 \mathrm{mg} /$ day produces significant cognitive, behavioral, and global improvements in DLB patients, with a relatively low discontinuation rate due to adverse events (AEs) [11].

Due to the progressive nature of DLB, it is clear that long-term treatment is essential; however, evidence of the long-term safety and efficacy of ChEIs has not been well established, as there is only one report assessing the long-term use of rivastigmine [8]. Also, worsening of parkinsonism and cardiac dysrhythmia are major concerns in the use of ChEIs. Patients with DLB may be more susceptible to bradyarrhythmic side effects due to the autonomic insufficiency associated with the disease [14]. Therefore, the benefits of long-term treatment with ChEIs in those patients remain an important clinical question.

Based on our findings from the preceding RCT, which suggested short-term benefits of treating DLB patients with donepezil, we designed an open-label extension study to investigate the safety and efficacy of long-term administration (52 weeks) of donepezil at $5 \mathrm{mg} /$ day in patients with DLB who had completed the preceding RCT.

This study was registered as No. NCT00598650.

\section{Patients and Methods}

Patients

Patients who satisfied the consensus diagnostic criteria for probable DLB [2] and who had completed the preceding phase 2, 12-week, randomized, double-blind, placebo-controlled study evaluating the efficacy and safety of donepezil [11] were subsequently recruited for this 52-week extension study. The study was conducted in 48 psychiatric and neurological specialty centers throughout Japan between February 2008 and March 2011.

Key inclusion and exclusion criteria for this extension study were the same as for the preceding RCT, and were described in detail in that study [11]. Briefly, patients diagnosed with probable DLB with mild to moderate-severe dementia and behavioral symptoms $[10 \leq$ Mini-Mental State Examination (MMSE) $\leq 26$, Neuropsychiatric Inventory (NPI) $\geq 8$ at baseline of the preceding RCT], aged $\geq 50$ years, were asked to participate in the study. Patients with conditions which might affect their cognitive functions, including focal vascular lesions and other neurological or psychiatric diseases, were excluded from this study. Patients who had severe extrapyramidal disorders (Hoehn \& Yahr staging $\geq$ IV), systolic blood pressure of $<90 \mathrm{~mm} \mathrm{Hg}$, pulse rate of $<50$ b.p.m., or QT interval prolongation (QTc $\geq 450 \mathrm{~ms}$ ) were also excluded. In order to collect reliable information about the patient's condition from his or her caregiver, patients were also required to have a reliable caregiver who spent at least $4 \mathrm{~h}$ per day with them for at least 3 days per week. 


\section{Study Design}

This was a 52-week, multicenter, open-label extension study to assess the long-term safety and efficacy of daily administration of $5 \mathrm{mg}$ of donepezil for the management of DLB. This study was a preplanned study, planned simultaneously with the preceding RCT. In the preceding RCT, patients were randomly assigned in a 1:1:1:1 ratio to receive a placebo or 3,5 , or $10 \mathrm{mg}$ of donepezil. Up to 8 weeks after the completion of the RCT, eligible patients who agreed to participate started the treatment period of this extension study with 3-mg doses of donepezil for 2 weeks, which was then increased to $5 \mathrm{mg}$ per day for the remaining 50 weeks. Dose adjustment to $3 \mathrm{mg}$ was permitted only when continuation of treatment with 5-mg doses was judged to be difficult due to AEs, in which case the dose was maintained throughout the remaining treatment period. During the transition period between the two studies, administration of donepezil was not allowed. In order to maintain blinding of the preceding RCT, the treatment with donepezil in this extension study was started after the data of each patient from the RCT had been fixed. It was not until 3 months after the last patient was enrolled in this study that the key code of the RCT was broken.

Written informed consent was again obtained from all caregivers and patients (if capable) before the start of this extension study. The study was conducted in accordance with the principles of the Declaration of Helsinki, and the study protocol was approved by the institutional review board of each participating center.

\section{Outcome Measures}

Cognitive function was assessed using the MMSE [15]. Behavioral and psychiatric symptoms of dementia and fluctuations in cognition were assessed using the NPI [16] and Cognitive Fluctuation Inventory (CFI), respectively. The CFI is a newly developed questionnaire to assess cognitive fluctuations in patients with dementia $[6,11]$. It employs the same format as the NPI, so as to enable practical comparison with the other symptoms assessed by the NPI. Its content validity has been assured based on reviews by experts, and reliability (both inter- and intrarater reliability) was demonstrated to be sufficient as a measure of cognitive fluctuation [unpubl. data]. These measures were assessed at 0, 4, 8, 16, 24, 32, 40, and 52 weeks. We also assessed caregiver burden using the Zarit Caregiver Burden Interview (ZBI) [17] at 0, 24, and 52 weeks.

Safety

All AEs, including abnormalities in vital signs, electrocardiograms, and laboratory tests, were recorded during the entire study period. The causal relationship to donepezil, severity, and outcome of each AE was assessed by attending physicians. For the assessment of parkinsonism, the patients' motor functions were assayed using the Unified Parkinson's Disease Rating Scale (UPDRS) part III [18].

\section{Statistical Analyses}

Safety analyses were performed on patients who received at least one dose of donepezil and who also provided safety assessment data after baseline. Of these patients, those with at least one available efficacy evaluation were included in efficacy analysis. Baseline demographic and clinical characteristics were summarized with descriptive statistics. For continuous variables, means and standard deviations were calculated. For categorical variables, the frequency of each category was calculated.

In this analysis, no formal primary endpoint was predefined due to the exploratory nature of this study. For safety, incidence rates of AEs were calculated by treatment period. The summary statistics of vital signs and UPDRS scores at each evaluation were also determined. For efficacy measures, mean change from baseline to each evaluation point was calculated. Mean change was also calculated by defining week 0 of the preceding RCT as baseline. These changes were calculated by treatment group in the preceding RCT. Values at the final evaluation were imputed using a last observation carried forward (LOCF) method. Statistical comparisons between baseline and each evaluation point were performed using paired t tests. All statistical tests were two tailed, and $\mathrm{p}<0.05$ was considered to indicate statistical significance.

\section{Results}

Baseline Characteristics

Out of 123 patients who had completed the RCT, 113 provided written informed consent to be screened. Of these, 108 patients who met the inclusion criteria were enrolled into the study performed at 40 sites. Two patients whose diagnosis was suspected not to meet clinical 


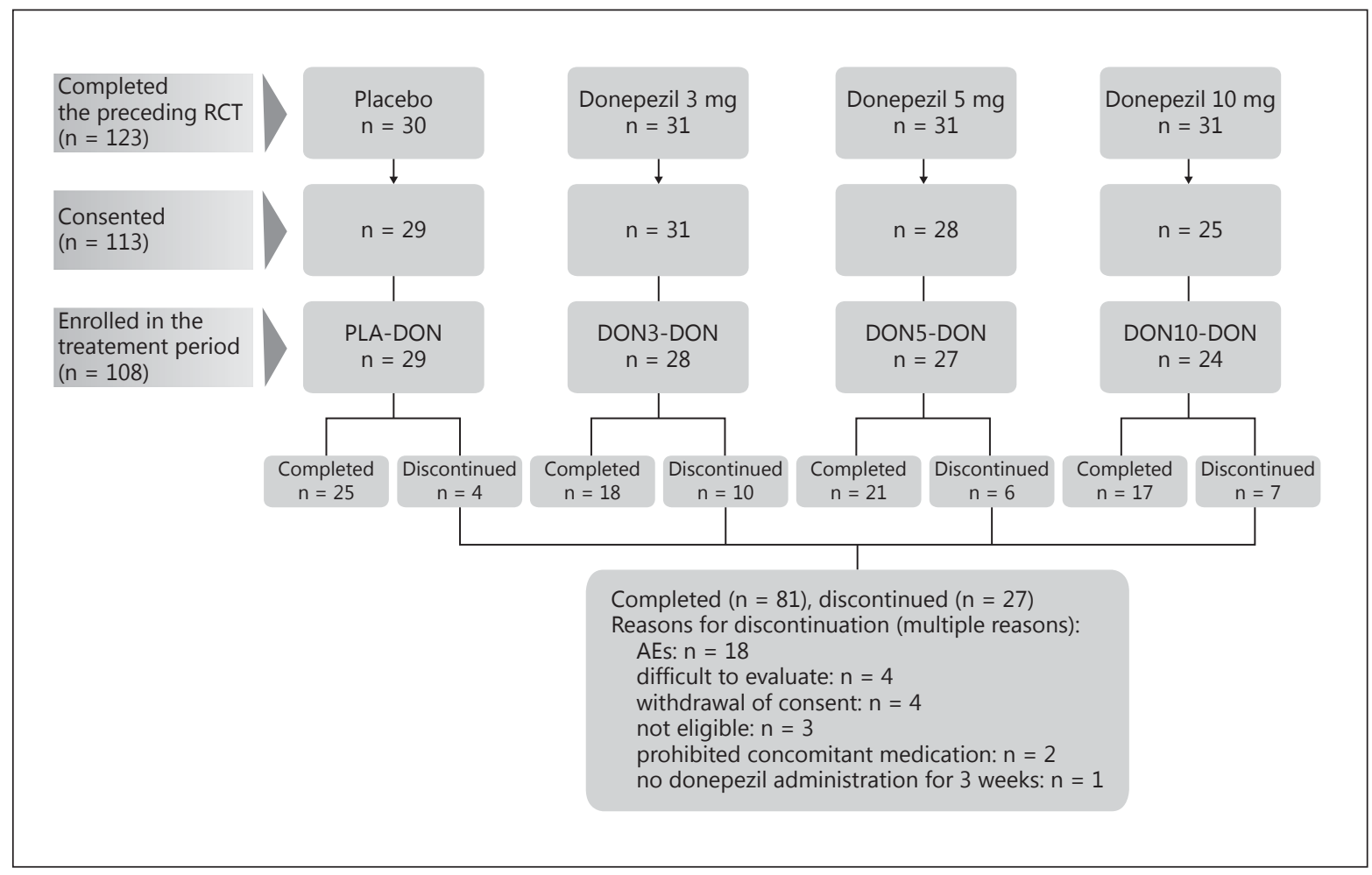

Fig. 1. Disposition of patients in the extension study.

criteria of probable DLB and another 2 patients with lack of efficacy data were excluded from the efficacy analysis population. The efficacy population $(n=104)$ consisted of 28 patients from the placebo group in the preceding RCT (referred as 'PLA-DON'), 27 patients from the donepezil 3-mg group (referred as 'DON3-DON'), 26 patients from the donepezil 5-mg group (referred as 'DON5-DON'), and 23 patients from the donepezil 10-mg group (referred as 'DON10-DON'; fig. 1). Baseline patient characteristics of the efficacy population are summarized in table 1. Mean scores of the MMSE, NPI-10, and the CFI at baseline were 20.9, 13.1, and 2.3 , respectively.

The mean transition period from the final administration in the preceding study to the initiation of this extension study was 12.6 days (SD: 5.8, range: 6-28). Mean changes in MMSE, $\mathrm{NPI}$, and CFI scores in the transition period by group are shown in table 2 . Attenuation in the treatment effect was observed in most of the DON-DON groups. Nevertheless, the MMSE scores in the DON5-DON and DON10-DON groups were still more than 3 points higher compared with the PLA-DON group, even after washout prior to the start of the extension study (table 1). Compared to the PLA-DON group, a better NPI score was still observed in the DON5-DON group (approximately 5 points better) and the DON10-DON group (approx. 7 points better) after the washout period (table 1). Compared with patients with a shorter washout period, those with a longer washout period ( 2 weeks or longer) were more likely to show deterioration in these measures.

Of the 108 patients enrolled in the study, 90 patients (83.3\%) completed 24 weeks (18 patients discontinued: 6 patients from 0 to 8 weeks, 6 patients from 8 to 16 weeks, and 6 patients from 16 to 24 weeks), and 81 patients (75\%) completed 52 weeks. The overall discontinuation rate of this study was $25 \%(\mathrm{n}=27)$, and 18 patients discontinued treatment 
Ikeda et al.: Long-Term Safety and Efficacy of Donepezil in Patients with Dementia with Lewy Bodies: Results from a 52-Week, Open-Label, Multicenter Extension Study

Table 1. Patient demographics and baseline characteristics of the efficacy population $(n=104)$

\begin{tabular}{|c|c|c|c|c|c|}
\hline & \multirow{2}{*}{$\begin{array}{l}\text { Overall } \\
(n=104)\end{array}$} & \multicolumn{4}{|c|}{ Treatment group in the preceding placebo-controlled study } \\
\hline & & $\begin{array}{l}\text { PLA-DON } \\
(\mathrm{n}=28)\end{array}$ & $\begin{array}{l}\text { DON3-DON } \\
(\mathrm{n}=27)\end{array}$ & $\begin{array}{l}\text { DON5-DON } \\
(n=26)\end{array}$ & $\begin{array}{l}\text { DON10-DON } \\
(\mathrm{n}=23)\end{array}$ \\
\hline Age, years & $79.1 \pm 5.7$ & $79.0 \pm 4.6$ & $80.3 \pm 4.8$ & $78.7 \pm 6.6$ & $78.2 \pm 6.6$ \\
\hline \multicolumn{6}{|l|}{ Sex, n (\%) } \\
\hline Male & $37(35.6)$ & $9(32.1)$ & $14(51.9)$ & $13(50.0)$ & $1(4.3)$ \\
\hline Female & $67(64.4)$ & $19(67.9)$ & $13(48.1)$ & $13(50.0)$ & $22(95.7)$ \\
\hline Weight, kg & $48.7 \pm 9.2$ & $48.0 \pm 8.3$ & $50.4 \pm 10.2$ & $50.6 \pm 8.8$ & $45.5 \pm 9.1$ \\
\hline \multicolumn{6}{|c|}{ Parkinsonism, n (\%) } \\
\hline Yes & $90(86.5)$ & $25(89.3)$ & $23(85.2)$ & $23(88.5)$ & $19(82.6)$ \\
\hline No & $14(13.5)$ & $3(10.7)$ & $4(14.8)$ & $3(11.5)$ & $4(17.4)$ \\
\hline \multicolumn{6}{|c|}{ Hoehn \& Yahr, n (\%) } \\
\hline I & $19(21.1)$ & $4(16.0)$ & $6(26.1)$ & $3(13.0)$ & $6(31.6)$ \\
\hline II & $34(37.8)$ & $7(28.0)$ & $10(43.5)$ & $10(43.5)$ & $7(36.8)$ \\
\hline III & $37(41.1)$ & $14(56.0)$ & $7(30.4)$ & $10(43.5)$ & $6(31.6)$ \\
\hline IV and V & 0 & 0 & 0 & 0 & 0 \\
\hline MMSE & $20.9 \pm 5.1$ & $18.6 \pm 4.3$ & $20.1 \pm 6.2$ & $23.1 \pm 3.5$ & $21.9 \pm 5.0$ \\
\hline NPI & $13.1 \pm 16.8$ & $15.5 \pm 13.4$ & $17.7 \pm 24.4$ & $10.3 \pm 15.4$ & $8.0 \pm 7.4$ \\
\hline CFI & $2.3 \pm 3.0$ & $3.3 \pm 2.7$ & $2.1 \pm 3.0$ & $2.2 \pm 3.4$ & $1.4 \pm 2.4$ \\
\hline ZBI & $24.7 \pm 15.7$ & $26.9 \pm 14.6$ & $26.6 \pm 18.1$ & $23.6 \pm 16.4$ & $21.1 \pm 13.3$ \\
\hline
\end{tabular}

Values are mean $\pm \mathrm{SD}$, unless otherwise specified.

Table 2. Mean change in MMSE, NPI, and CFI from the end of the preceding RCT to the start of this extension study by treatment group in the preceding RCT

\begin{tabular}{|c|c|c|c|c|c|c|}
\hline \multirow{3}{*}{$\begin{array}{l}\text { Scores by } \\
\text { treatment group }\end{array}$} & \multirow{2}{*}{\multicolumn{2}{|c|}{ Overall }} & \multicolumn{4}{|c|}{ Washout period } \\
\hline & & & \multicolumn{2}{|c|}{$<2$ weeks } & \multicolumn{2}{|c|}{$\geq 2$ weeks } \\
\hline & $\mathrm{n}$ & mean \pm SD & $\mathrm{n}$ & mean \pm SD & $\mathrm{n}$ & mean $\pm S D$ \\
\hline \multicolumn{7}{|l|}{ MMSE } \\
\hline PLA-DON & 27 & $0.2 \pm 2.2$ & 14 & $-0.1 \pm 2.0$ & 13 & $0.5 \pm 2.5$ \\
\hline DON3-DON & 27 & $-0.9 \pm 3.0$ & 12 & $-0.5 \pm 2.2$ & 15 & $-1.2 \pm 3.5$ \\
\hline DON5-DON & 26 & $-0.8 \pm 2.7$ & 12 & $0.4 \pm 2.4$ & 14 & $-1.9 \pm 2.5$ \\
\hline DON10-DON & 22 & $0.1 \pm 2.7$ & 14 & $0.7 \pm 2.7$ & 8 & $-1.0 \pm 2.4$ \\
\hline \multicolumn{7}{|l|}{ NPI } \\
\hline PLA-DON & 28 & $-0.3 \pm 5.1$ & 14 & $-1.3 \pm 4.3$ & 14 & $0.7 \pm 5.7$ \\
\hline DON3-DON & 27 & $1.9 \pm 8.4$ & 12 & $-0.3 \pm 5.0$ & 15 & $3.6 \pm 10.1$ \\
\hline DON5-DON & 26 & $3.7 \pm 12.5$ & 12 & $8.3 \pm 15.7$ & 14 & $-0.1 \pm 7.5$ \\
\hline DON10-DON & 21 & $0.3 \pm 4.8$ & 14 & $-0.5 \pm 4.3$ & 7 & $2.0 \pm 5.7$ \\
\hline \multicolumn{7}{|l|}{ CFI } \\
\hline PLA-DON & 28 & $0.1 \pm 2.0$ & 14 & $0.0 \pm 1.4$ & 14 & $0.2 \pm 2.6$ \\
\hline DON3-DON & 27 & $-0.2 \pm 1.8$ & 12 & $-1.1 \pm 1.5$ & 15 & $0.5 \pm 1.8$ \\
\hline DON5-DON & 26 & $0.7 \pm 2.2$ & 12 & $1.3 \pm 2.9$ & 14 & $0.1 \pm 1.1$ \\
\hline DON10-D0N & 21 & $-0.2 \pm 1.9$ & 14 & $-0.4 \pm 2.2$ & 7 & $0.0 \pm 1.3$ \\
\hline
\end{tabular}

The negative MMSE scores, the positive NPI scores, and the positive CFI scores indicate deterioration. 


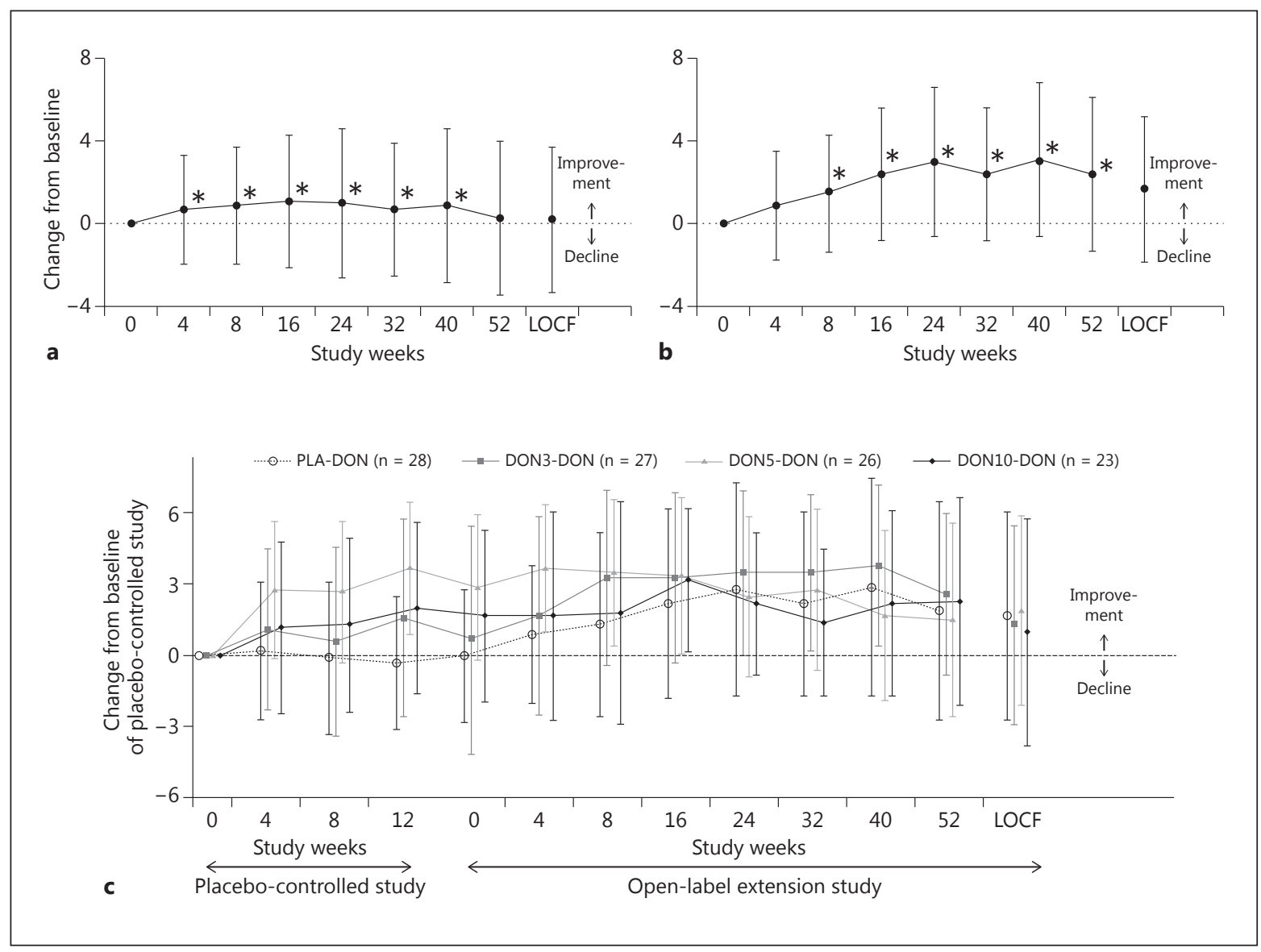

Fig. 2. Mean changes in MMSE scores. ${ }^{*} \mathrm{p}<0.05$ vs. baseline (paired t test). Vertical bars indicate standard deviations. a Overall mean change during the treatment period $(n=103)$. b Mean change in the placebo group of the preceding RCT $(n=27)$. c Mean cumulative changes by treatment group in the preceding RCT throughout both the preceding RCT and this extension study (no statistical test was performed).

due to AEs (fig. 1). Three patients underwent a dose reduction from 5 to $3 \mathrm{mg} /$ day due to the occurrence of AEs. Of these, 2 patients completed the study with a dose of $3 \mathrm{mg} /$ day without premature termination of the study, despite having an AE occur even after the dose reduction.

\section{Efficacy}

Mean scores in MMSE significantly improved at 4-40 weeks compared with baseline (fig. 2a). The mean (SD) changes at 52 weeks and at the final evaluation (LOCF) from baseline were $0.3 \pm 3.7$ and $0.2 \pm 3.5$, respectively, indicating that baseline values were maintained over 52 weeks. In the PLA-DON group (fig. 2b), the mean (SD) changes at 52 weeks and at the final evaluation (LOCF) from baseline were $2.0 \pm 4.4$ and $1.7 \pm 4.4$, respectively, and the largest change was observed at 40 weeks $(3.0 \pm 4.3)$. Significant improvement was demonstrated at all the evaluation points after 8 weeks. If the results are analyzed by defining week 0 of the previous RCT as baseline, although there was up to an 8-week washout period, MMSE scores in DON-DON groups improved throughout the RCT and the subsequent 52-week extension study (longer than 64 weeks in total; fig. 2c). On the other hand, in the PLA-DON group, improvement in MMSE scores was found only during the extension period. 


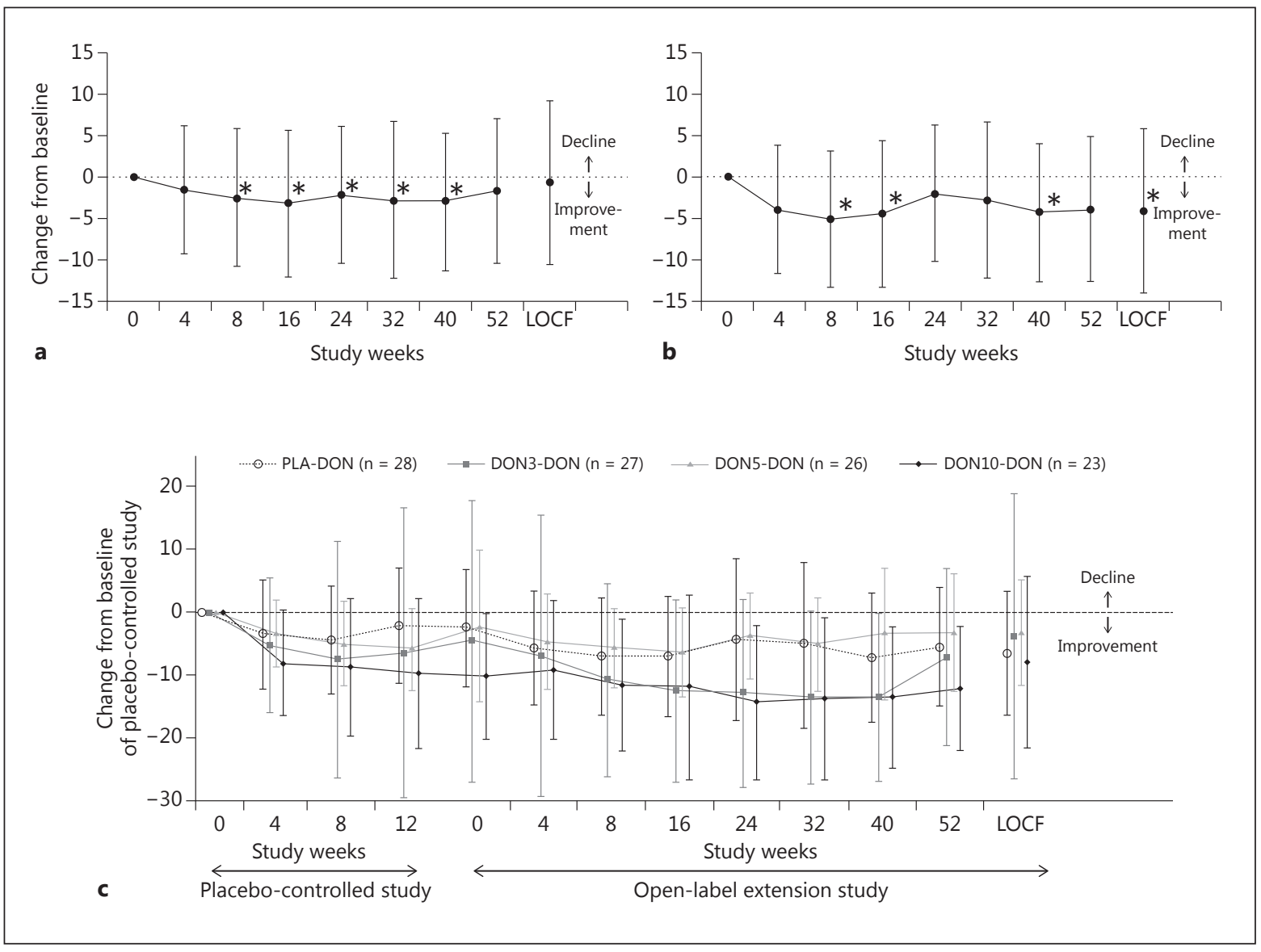

Fig. 3. Mean changes in NPI scores. ${ }^{*} p<0.05$ vs. baseline (paired t test). Vertical bars indicate standard deviations. a Overall mean change during the treatment period $(n=104)$. $\mathbf{b}$ Mean change in the placebo group in the preceding RCT $(\mathrm{n}=28)$. c Mean cumulative changes by treatment group in the preceding RCT throughout both the preceding RCT and this extension study (no statistical test was performed).

Changes in NPI scores during the treatment period are shown in figure 3a. The mean (SD) changes at 52 weeks and at the final evaluation (LOCF) from baseline were $-1.9 \pm 9.8$ and -0.7 \pm 11.1 , respectively. A significant improvement in the scores compared to baseline was observed at $8-40$ weeks, and the largest change was observed at 16 weeks $(-3.6 \pm 9.9)$. In the PLA-DON group, significant improvement was demonstrated at 8, 16, and 40 weeks, and at the final evaluation point (LOCF; fig. 3b). The mean changes at 52 weeks and at the final evaluation point (LOCF) were $-4.1 \pm 10.1$ and $-4.3 \pm 9.7$, respectively. During the entire treatment period, combining both the preceding study and this extension study, improvement was maintained in all the DON-DON groups, even though there was up to an 8-week washout period (fig. 3c).

The changes in CFI scores during the treatment period are shown in figure 4a. Significant improvement compared to baseline was observed at 24 and 32 weeks, and this improvement was maintained throughout the treatment period. In the PLA-DON group, significant improvement was demonstrated at 8 and 16 weeks (fig. 4b). The mean (SD) changes at 52 weeks and at the final evaluation point (LOCF) were $-1.0 \pm 2.7$ and $-1.0 \pm 2.6$, respectively, and the largest change was observed at 8 weeks $(-1.4 \pm 1.8)$. If the results are analyzed by defining week 0 of the preceding RCT as baseline, CFI scores in all the DON-DON 


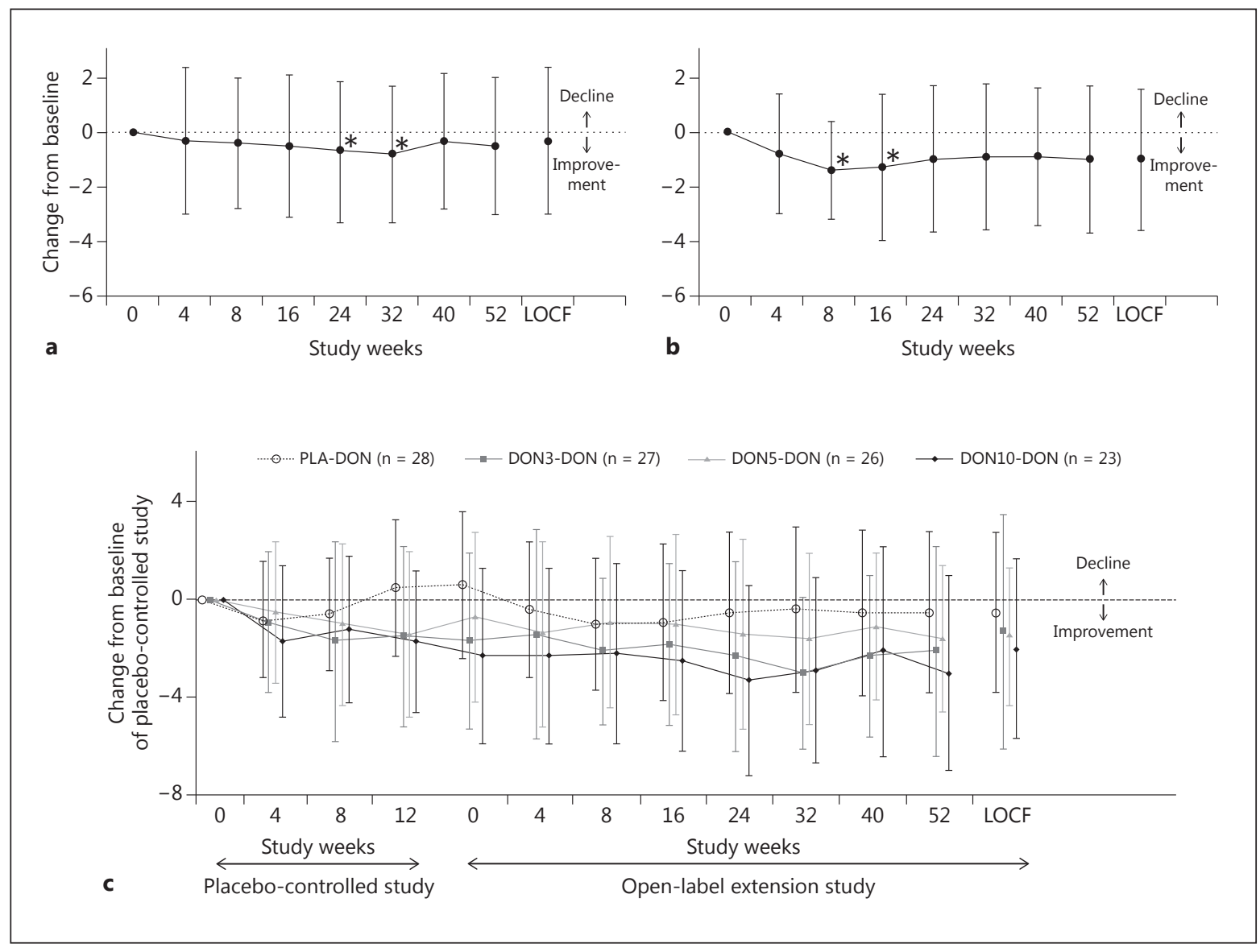

Fig. 4. Mean changes in CFI scores. ${ }^{*} p<0.05$ vs. baseline (paired t test). Vertical bars indicate standard deviations. a Overall mean change during the treatment period $(n=104)$. $\mathbf{b}$ Mean change in the placebo group in the preceding RCT $(n=28)$. c Mean cumulative changes by treatment group in the preceding RCT throughout both the preceding RCT and this extension study (no statistical test was performed).

groups improved throughout the RCT and the subsequent 52-week extension study (fig. 4c).

With regard to caregiver burden, a significant deterioration was demonstrated at 52 weeks and at the final evaluation point (LOCF) compared to baseline (fig. 5a). In the PLA-DON group, however, improvements in scores were observed during the treatment period, although they were not statistically significant (fig. 5b). In the DON-DON groups, ZBI scores were likely to be improved during the preceding RCT; however, this degree of improvement disappeared during the extension period (fig. 5c).

\section{Safety}

The incidence of AEs in the safety analysis set was $94.4 \%(102 / 108)$. Incidence rates of AEs did not differ among the four groups stratified according to the preceding RCT $189.7 \%$ in PLA-DON, 96.4\% in DON3-DON, 92.6\% in DON5-DON, and 100.0\% in DON10-DON). Twentyseven serious AEs were reported in 25 patients. Serious AEs observed in more than 1 patient included compression fractures $(n=3)$, pneumonia $(n=3)$, and dehydration $(n=2)$. Four events (myocardial infarction, subarachnoid hemorrhage, asphyxia, and acute pancreatitis) resulted in the deaths of 3 patients. Myocardial infarction and acute pancreatitis were deter- 


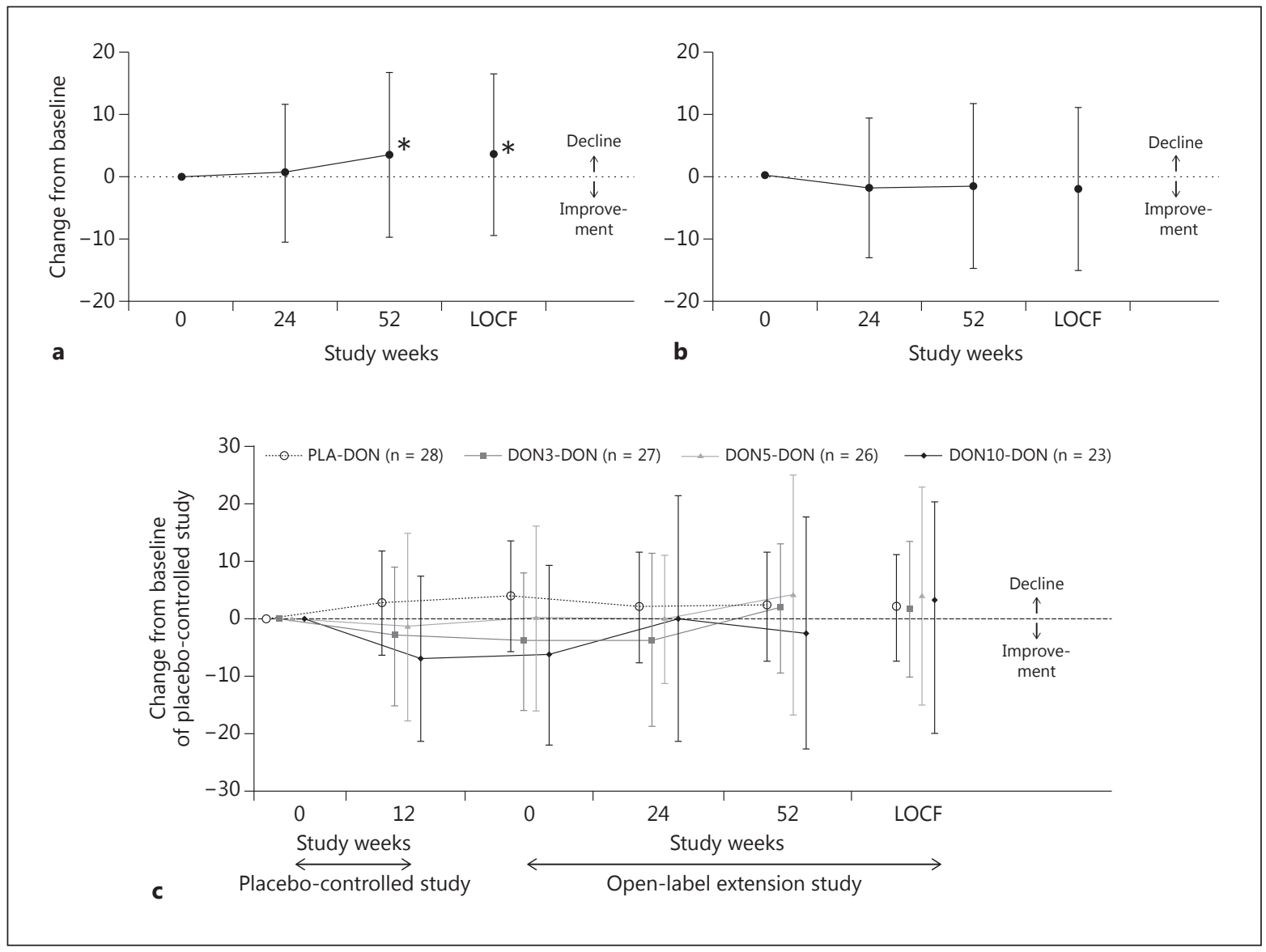

Fig. 5. Mean changes in ZBI scores. * $p<0.05$ vs. baseline (paired t test). Vertical bars indicate standard deviations. a Overall mean change during the treatment period $(n=103)$. $\mathbf{b}$ Mean change in the placebo group in the preceding RCT $(n=28)$. c Mean cumulative changes by treatment group in the preceding RCT throughout both the preceding RCT and this extension study (no statistical test was performed).

mined to be the results of adverse drug reactions. Major frequently observed AEs are shown in table 3. They included increased blood creatine phosphokinase and contusion (12 patients each, 11.1\%), followed by nasopharyngitis, blood pressure increase, fall (11 patients each, $10.2 \%$ ), and diarrhea (10 patients, 9.3\%).

AEs associated with parkinsonism were reported in $12.0 \%(n=13)$ of the patients throughout the study period. Most of them (12/13) were mild to moderate in severity. No notable increase in these AEs was observed according to the treatment period. A modest and insignificant rise in the mean UPDRS score (range: 0.5-1.1) was noted at 24 and 52 weeks, and at the final evaluation point (LOCF).

The incidence of abnormal changes in pulse rate and abnormal electrocardiograms was 1.9 ( 2 patients) and $7.5 \%$ ( 8 patients), respectively. The mean pulse rate decreased modestly at every evaluation point compared to baseline (range: -0.3 to -1.8 ). AEs related to them included prolonged QT (2 patients), supraventricular extrasystoles (2 patients), first-degree atrioventricular block (1 patient), bradycardia (1 patient), sinus bradycardia (1 patient), and ventricular extrasystoles (1 patient) on electrocardiogram. None of these events was serious or clinically significant (e.g., leading to discontinuation or dose reduction). 
Ikeda et al.: Long-Term Safety and Efficacy of Donepezil in Patients with Dementia with Lewy Bodies: Results from a 52-Week, Open-Label, Multicenter Extension Study

Table 3. AEs $[\mathrm{n}(\%)]$ reported by $\geq 5 \%$ of patients during the entire period (all causality)

\begin{tabular}{|c|c|c|c|c|}
\hline & \multirow{2}{*}{$\begin{array}{l}\text { Overall } \\
(n=108)\end{array}$} & \multicolumn{3}{|l|}{ Time of onset } \\
\hline & & $\begin{array}{l}0-12 \text { weeks } \\
(\mathrm{n}=108)\end{array}$ & $\begin{array}{l}12-24 \text { weeks } \\
(\mathrm{n}=98)\end{array}$ & $\begin{array}{l}24-52 \text { weeks } \\
(\mathrm{n}=90)\end{array}$ \\
\hline Blood CPK increase & $12(11.1)$ & $8(7.4)$ & $3(3.1)$ & $3(3.3)$ \\
\hline Contusion & $12(11.1)$ & $4(3.7)$ & $5(5.1)$ & $4(4.4)$ \\
\hline Nasopharyngitis & $11(10.2)$ & $4(3.7)$ & $4(4.1)$ & $3(3.3)$ \\
\hline Blood pressure increase & $11(10.2)$ & $8(7.4)$ & $3(3.1)$ & $1(1.1)$ \\
\hline Fall & $11(10.2)$ & $5(4.6)$ & $1(1.0)$ & $7(7.8)$ \\
\hline Diarrhea & $10(9.3)$ & $3(2.8)$ & $5(5.1)$ & $3(3.3)$ \\
\hline Constipation & $8(7.4)$ & $2(1.9)$ & $4(4.1)$ & $2(2.2)$ \\
\hline Parkinsonism & $8(7.4)$ & $1(0.9)$ & $2(2.0)$ & $6(6.7)$ \\
\hline Blood urine present & $7(6.5)$ & $2(1.9)$ & $3(3.1)$ & $3(3.3)$ \\
\hline Protein urine present & $7(6.5)$ & $2(1.9)$ & $2(2.0)$ & $4(4.4)$ \\
\hline Decreased appetite & $6(5.6)$ & $1(0.9)$ & $1(1.0)$ & $4(4.4)$ \\
\hline Insomnia & $6(5.6)$ & $2(1.9)$ & $1(1.0)$ & $3(3.3)$ \\
\hline Compression fracture & $6(5.6)$ & $2(1.9)$ & 0 & $4(4.4)$ \\
\hline
\end{tabular}

$\mathrm{CPK}=$ Creatine phosphokinase .

AEs associated with gastrointestinal symptoms were reported in 34 patients $(31.5 \%)$. Diarrhea (10 patients, $9.3 \%$ ), constipation (8 patients, $7.4 \%$ ), and decreased appetite (6 patients, 5.6\%) were observed relatively frequently.

AEs associated with psychiatric symptoms were noted in 25 patients $(23.1 \%)$. Six patients (5.6\%) experienced insomnia. Visual hallucinations and psychiatric symptoms were recorded as AEs in 5 patients (4.6\%), respectively. Incidence rates by onset time did not reveal any notable imbalance.

\section{Discussion}

This is the first study to examine long-term safety and efficacy of donepezil in patients with DLB. Overall, 108 patients with DLB who had completed the 12-week, double-blind, comparative RCT subsequently participated in this extension study. The results presented here demonstrate that cognitive function and dementia-related behavioral symptoms, including cognitive fluctuations, were improved after the start of donepezil treatment, and maintained for 52 weeks, or up to 64 weeks if the preceding treatment period is included. Our findings suggest that treatment efficacy of donepezil for these symptoms may be maintained even after the treatment in patients who were followed in this extension study, since no linear decrease in evaluation scores was observed. In accordance with our results, the study of longterm use of rivastigmine in DLB patients revealed that the reduction in MMSE scores was gradual and without statistical significance compared to baseline for 96 weeks [8]. Additionally, no significant worsening of NPI scores was demonstrated, although the decline in the scores seemed sharper after 72 weeks [8]. In contrast, when donepezil was administered to patients with $\mathrm{AD}$ for 52 weeks, it was reported that cognitive function, as assessed by the MMSE or Severe Impairment Battery [19], started to decline after 24 weeks [20, 21]. Progression of cognitive impairment in DLB and AD patients has been compared in several studies, but results differ from study to study. Olichney et al. [22] reported that there was a significant difference between DLB and AD groups in mean MMSE decline per year $(-5.8 \pm 4.5$ 
for the DLB group and $-4.1 \pm 3.0$ for the AD group). Ballard et al. [23] reported that more deterioration in the mean MMSE score per year was observed in an AD group $(-4.9 \pm 3.6)$ than a DLB group $(-4.3 \pm 4.2)$, although no statistical difference was shown. Furthermore, a similar cognitive decline between a DLB and an AD group was reported by Walker et al. [24]. The mean decline in the MMSE score per year was $-3.1 \pm 4.3$ for the DLB group and $-2.6 \pm 4.0$ for the AD group, with no statistically significant difference. These results indicate that cognitive decline in DLB may be faster than or at least similar to that in AD patients, and, in this respect, patients with DLB might be more likely to benefit from donepezil treatment compared to AD patients. With regard to burden on caregivers, no obvious improvement was shown in ZBI scores, while the treatment effect on cognitive functions, as well as neuropsychiatric symptoms, were improved or at least maintained. Accumulation of caregiving burden over time may prevent caregivers from realizing that a decrease in burden has occurred. However, it is noteworthy that burden on caregivers did not increase throughout the cumulative observational period in our two studies.

Unsurprisingly, a relationship between the washout period and attenuation in the treatment effect was suggested. Among patients who were assigned to the donepezil treatment groups in the preceding RCT, cognitive function and behavioral/psychiatric symptoms deteriorated more in patients with a longer washout period. This could indicate that the treatment effect might eventually diminish if donepezil administration was stopped for a long period of time.

Since there was no significant imbalance in the AE incidence analyzed by onset time, it is therefore suggested that delayed onset of AE induced by long-term donepezil administration is unlikely to appear in these patients. Patients with DLB may be at increased risk of bradyarrhythmia resulting from treatment with ChEIs though [14]. In this long-term study, however, only 2 patients experienced abnormal changes in pulse rate 1 bradycardia and 1 sinus bradycardia), and neither of these were serious. Also, long-term administration of donepezil is unlikely to worsen parkinsonian symptoms since UPDRS scores did not worsen over 52 weeks. Furthermore, only 3 patients received dose reductions to $3 \mathrm{mg} /$ day due to AEs. Two of them completed this study with the reduced dose, thereby enabling the patients to continue treatment with donepezil by reducing the dosage to $3 \mathrm{mg} /$ day. In comparison to a study of donepezil in patients with $\mathrm{AD}$, AEs reported in this study were similar to those reported in the study of AD patients, except for parkinsonism [20].

The major limitation of this study is its open-label, single-arm design. Clearly, a blinded, comparative study is necessary to confirm our findings; however, due to the progressive nature of this disease, leading to acceleration of mortality, allocating patients to a placebo is not appropriate for long periods of time. Because improvement in MMSE scores and NPI scores after donepezil administration in the PLA-DON group showed a similar trend with the results presented in the preceding double-blind RCT, despite the open-label design used in this study, we believe that our results reliably indicate the efficacy of donepezil. It should also be noted that this study cannot determine which donepezil dose might contribute to a better outcome on a long-term basis. Since the preceding RCT suggested the benefit of the administration of a 10-mg dose in a particular group of patients, compared to 3 or $5 \mathrm{mg}$, further research would be helpful to assess the long-term benefits of administration of 10-mg doses.

In conclusion, the long-term administration of donepezil at $5 \mathrm{mg}$ /day was safe in patients with DLB, and is expected to exhibit lasting effects on improving impaired cognitive function and psychiatric symptoms. 
Ikeda et al.: Long-Term Safety and Efficacy of Donepezil in Patients with Dementia with Lewy Bodies: Results from a 52-Week, Open-Label, Multicenter Extension Study

\section{Acknowledgments}

We are deeply grateful for the participation of the patients and their caregivers. We also thank all investigators and site staff for their contributions, and the Eisai study team (M. Hayashi, T. Kobayashi, S. Taniguchi, and E. Ebisawa) for their assistance. This study was sponsored by Eisai Co., Ltd. (Tokyo, Japan).

\section{Donepezil-DLB Study Investigators}

Sadao Katayama (Hiroshima-nishi Medical Center), Yasuto Higashi (Himeji Central Hospital), Tatsuo Yamada (Fukuoka University Hospital), Yuichi Maruki (Saitama Neuropsychiatric Institute), Satoshi Orimo (Kanto Central Hospital), Aoi Yoshiiwa (Oita University Hospital), Haruo Hanyu (Tokyo Medical University Hospital), Masayuki Yokochi (Ebara Hospital), Takemi Kimura (Kikuti National Hospital), Koichi Mizoguchi (Shizuoka Institute of Epilepsy and Neurology), Aki Nakanishi (Osaka City Kosaiin Hospital), Tadashi Tsukamoto (National Center of Neurology and Psychiatry), Norio Taniguchi (Asakayama General Hospital), Koichi Okamoto (Gunma University Hospital), Tatsuru Kitamura (Takamatsu Hospital), Yoko Nakano (Sukoyaka-Silver Hospital), Tomonobu Kato (Osaka Red Cross Hospital), Kenichi Shimada (Hyogo Brain and Heart Center), Masanori Hiji (Vi-hara Hananosato Hospital), Yasumasa Yoshiyama (Chiba-East Hospital), Yuri Kitamura (Nanohana Clinic), Satoshi Takahashi (Iwate Medical University Hospital), Masahiro Akishita (Tokyo University Hospital), Yukihiko Washimi (National Center for Geriatrics and Gerontology), Yasuji Yamamoto (Kobe University Hospital), Miyuki Kobayashi (Komoro Kogen Hospital), Fukashi Udaka (Sumitomo Hospital), Yasushi Osaki (Kochi University Hospital), Hiroaki Hino (Yokohama Hoyu Hospital), Takashi Kanda (Yamaguchi University Hospital), Toshifumi Kishimoto (Nara Medical University Hospital), Hiroaki Oguro (Shimane University Hospital), Toshimasa Matsuoka (Kurume University Hospital), Yasuhiro Tsugu (Toyokawa City Hospital), Naoki Fujii (Omuta Hospital), and Yasuhiro Kawase (Kawase Neurology Clinic).

\section{Disclosure Statements}

M.I.: grants/grants pending, Daiichi Sankyo, Eisai, FUJIFILM RI, Janssen, Nihonmediphysics, Novartis, Pfizer, Takeda, Tsumura; speaking fees, Daiichi Sankyo, Eisai, FUJIFILM RI, Janssen, MSD, Nihonmediphysics, Novartis, Ono, Pfizer, Takeda, Tsumura.

E.M.: consultancy, Lundbeck; grants/grants pending, Eisai, FUJIFILM RI, Nihonmediphysics; speaking fees, Eisai, FUJIFILM RI, Janssen, Johnson \& Johnson, Lundbeck, Nihonmediphysics, Novartis.

K.K.: board membership, Dainippon Sumitomo, Eisai, Novartis, Tsumura; speaking fees, Daiichi Sankyo, Eisai, FUJIFILM RI, Janssen, Novartis, Nihonmediphysics, Ono, Pfizer, Tsumura; paid manuscript preparation, Tsumura.

E.I.: grants/grants pending, Daiichi Sankyo, Eisai, Nihonmediphysics, Novartis, Ono, Takeda, Tsumura; speaking fees, Daiichi Sankyo, Eisai, Janssen, Nihonmediphysics, Novartis, Ono, Takeda.

M.H.: speaking fees, Daiichi Sankyo, Eisai, FUJIFILM RI, Janssen, Nihonmediphysics, Novartis, Pfizer.

N.M.: grants/grants pending, Daiichi Sankyo.

K.M., M.N.: employees of Eisai.

\section{References}

1 McKeith I, Mintzer J, Aarsland D, Burn D, Chiu H, Cohen-Mansfield J, Dickson D, Dubois B, Duda JE, Feldman H, Gauthier S, Halliday G, Lawlor B, Lippa C, Lopez OL, Carlos Machado J, O’Brien J, Playfer J, Reid W: International Psychogeriatric Association Expert Meeting on DLB. Dementia with Lewy bodies. Lancet Neurol 2004;3: 19-28.

2 McKeith IG, Galasko D, Kosaka K, Perry EK, Dickson DW, Hansen LA, Salmon DP, Lowe J, Mirra SS, Byrne EJ, Lennox G, Quinn NP, Edwardson JA, Ince PG, Bergeron C, Burns A, Miller BL, Lovestone S, Collerton D, Jansen EN, Ballard C, de Vos RA, Wilcock GK, Jellinger KA, Perry RH: Consensus guidelines for the clinical and pathologic diagnosis of dementia with Lewy bodies (DLB): report of the consortium on DLB international workshop. Neurology 1996;47:1113-1124.

3 Perry EK, Haroutunian V, Davis KL, Levy R, Lantos P, Eagger S, Honavar M, Dean A, Griffiths M, McKeith IG, et al: Neocortical cholinergic activities differentiate Lewy body dementia from classical Alzheimer's disease. Neuroreport 1994;5:747-749. 
Ikeda et al.: Long-Term Safety and Efficacy of Donepezil in Patients with Dementia with Lewy Bodies: Results from a 52-Week, Open-Label, Multicenter Extension Study

- 4 Perry EK, Irving D, Kerwin JM, McKeith IG, Thompson P, Collerton D, Fairbairn AF, Ince PG, Morris CM, Cheng $\mathrm{AV}$, et al: Cholinergic transmitter and neurotrophic activities in Lewy body dementia: similarity to Parkinson's and distinction from Alzheimer disease. Alzheimer Dis Assoc Disord 1993;7:69-79.

5 Thomas AJ, Burn DJ, Rowan EN, Littlewood E, Newby J, Cousins D, Pakrasi S, Richardson J, Sanders J, McKeith IG: A comparison of the efficacy of donepezil in Parkinson's disease with dementia and dementia with Lewy bodies. Int J Geriatr Psychiatry 2005;20:938-944.

6 Mori S, Mori E, Iseki E, Kosaka K: Efficacy and safety of donepezil in patients with dementia with Lewy bodies: preliminary findings from an open-label study. Psychiatry Clin Neurosci 2006;60:190-195.

- 7 Rowan E, McKeith IG, Saxby BK, O’Brien JT, Burn D, Mosimann U, Newby J, Daniel S, Sanders J, Wesnes K: Effects of donepezil on central processing speed and attentional measures in Parkinson's disease with dementia and dementia with Lewy bodies. Dement Geriatr Cogn Disord 2007;23:161-167.

8 Grace J, Daniel S, Stevens T, Shankar KK, Walker Z, Byrne EJ, Butler S, Wilkinson D, Woolford J, Waite J, McKeith IG: Long-term use of rivastigmine in patients with dementia with Lewy bodies: an open-label trial. Int Psychogeriatr 2001;13:199-205.

9 Edwards K, Royall D, Hershey L, Lichter D, Hake A, Farlow M, Pasquier F, Johnson S: Efficacy and safety of galantamine in patients with dementia with Lewy bodies: a 24-week open-label study. Dement Geriatr Cogn Disord 2007;23:401-405.

10 McKeith I, Del Ser T, Spano P, Emre M, Wesnes K, Anand R, Cicin-Sain A, Ferrara R, Spiegel R: Efficacy of rivastigmine in dementia with Lewy bodies: a randomised, double-blind, placebo-controlled international study. Lancet 2000;356:2031-2036.

11 Mori E, Ikeda M, Kosaka K: Donepezil-DLB Study Investigators. Donepezil for dementia with Lewy bodies: a randomized, placebo-controlled trial. Ann Neurol 2012;72:41-52.

12 Emre M, Aarsland D, Albanese A, Byrne EJ, Deuschl G, De Deyn PP, Durif F, Kulisevsky J, van Laar T, Lees A, Poewe W, Robillard A, Rosa MM, Wolters E, Quarg P, Tekin S, Lane R: Rivastigmine for dementia associated with Parkinson's disease. N Engl J Med 2004;351:2509-2518.

13 Dubois B, Tolosa E, Katzenschlager R, Emre M, Lees AJ, Schumann G, et al: Donepezil in Parkinson's disease dementia: a randomized, double-blind efficacy and safety study. Mov Disord 2012;27:1230-1238.

14 Rosenbloom MH, Finley R, Scheinman MM, Feldman MD, Miller BL, Rabinovici GD: Donepezil-associated bradyarrhythmia in a patient with dementia with Lewy bodies (DLB). Alzheimer Dis Assoc Disord 2010;24: 209-211.

15 Folstein MF, Folstein SE, McHugh PR: 'Mini-mental state'. A practical method for grading the cognitive state of patients for the clinician. J Psychiatr Res 1975;12:189-198.

-16 Cummings JL, Mega M, Gray K, Rosenberg-Thompson S, Carusi DA, Gornbein J: The Neuropsychiatric Inventory: comprehensive assessment of psychopathology in dementia. Neurology 1994;44:2308-2314.

-17 Zarit SH, Reever KE, Bach-Peterson J: Relatives of the impaired elderly: correlates of feelings of burden. Gerontologist 1980;20:649-655.

18 Fahn S, Elton RL; UPDRS Development Committee: Unified Parkinson's Disease Rating Scale; in Fahn S, Marsden CD, Calne D, Goldstein M (eds): Recent Developments in Parkinson's Disease. Florham Park, Macmillan Healthcare Information, 1987, vol 2, pp 153-163, 293-304.

19 Panisset M, Roudier M, Saxton J, Boller F: Severe impairment battery. A neuropsychological test for severely demented patients. Arch Neurol 1994;51:41-45.

20 Tohgi H, Homma A, Imai Y, Udaka F, Takeda M, Nishimura T, Kameyama M, Hasegawa K: Long-term safety and efficacy of acetylcholinesterase inhibitor E2020 in patients with Alzheimer-type dementia. 52-week open label study. Clin Eval 2000;28:97-126.

-21 Homma A, Imai Y, Tago H, Asada T, Shigeta M, Iwamoto T, Takita M, Arimoto I, Koma H, Takase T, Ohbayashi T: Long-term safety and efficacy of donepezil in patients with severe Alzheimer's disease: results from a 52-week, open-label, multicenter, extension study in Japan. Dement Geriatr Cogn Disord 2009;27:232-239.

22 Olichney JM, Galasko D, Salmon DP, Hofstetter CR, Hansen LA, Katzman R, Thal LJ: Cognitive decline is faster in Lewy body variant than in Alzheimer's disease. Neurology 1998;51:351-357.

23 Ballard C, O’Brien J, Morris CM, Barber R, Swann A, Neill D, McKeith I: The progression of cognitive impairment in dementia with Lewy bodies, vascular dementia and Alzheimer's disease. Int J Geriatr Psychiatry 2001;16: 499-503.

24 Walker Z, McKeith I, Rodda J, Qassem T, Tatsch K, Booij J, Darcourt J, O’Brien J: Comparison of cognitive decline

between dementia with Lewy bodies and Alzheimer's disease: a cohort study. BMJ Open 2012;2:e000380. 